\title{
High-Speed Response of Liquild Crystals Using Polymer Stabilization
}

\author{
Kimio Hibi, Shunsuke Kobayashi* and Hirokazu Furue \\ Tokyo University of Science, Noda, Chiba 278-8510, Japan \\ *Tokyo University of Science-Yamaguchi, Sanyouonoda, Yamaguchi 756-0884, Japan
}

\begin{abstract}
The polymer stabilization is an attractive technique because the physical property of liquid crystals can be improved and furthermore the new characteristics may occur. In this study, we try to obtain a high-speed response of nematic liquid crystals by using polymer stabilization technique for realization of the field sequential color. We have researched the dependence of the electrooptical characteristics such as response time and threshold voltage on UV irradiation energy, polymer concentration and temperature at polymerization. As a result, it is found that the electrooptical characteristics strongly depend on their conditions.
\end{abstract}

Keywords: liquid crystal, polymer stabilization, response time, threshold voltage, field sequential color

\section{Introduction}

Currently, the color filter (CF) system is used for the color expression of liquid crystal displays (LCDs) which are available in the market. In the CF system, a pixel consists of three sub-pixels with red, green and blue color (RGB) (Fig. 1). Therefore, the energy efficiency to the incident white light does not exceed 1/3, and the black matrix reduces further the efficiency. Furthermore, in the CF system, the micro-fabrication technique is necessary to divide a pixel into three sub-pixels.

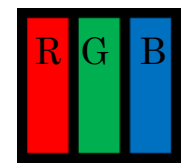

Fig. 1 CF system (a pixel)

On the other hand, the field sequential color (FSC) system is expected for a next generation LCD. The FSC system is based on the temporal color method in which RGB color backlights are turned on sequentially and LC device is used as an optical shutter (Fig. 2) [1-5]. In comparison to conventional LCDs which use CF, the FSC-LCDs have the following potential advantages since no
$\mathrm{CF}$ is used: high luminance, low power consumption, high resolution, simplicity of fabrication, and simplicity of the driving circuit. In order to realize the FSC system, the response time of liquid crystal must be less than 1/180 seconds for $1 / 60$ seconds of 1 picture frame. Moreover, for suppressing color brake, the response time should be decreased to sub-millisecond. However, the response time of conventional nematic LCDs is about $10 \mathrm{~ms}$.

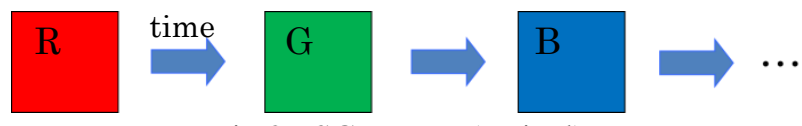

Fig.2 FSC system (a pixel)

In this research, we try to realize a high-speed response of nematic LCs by using a polymer stabilization technique. The polymer stabilization, in which a photocurable mesogenic monomer is doped into a LC medium and a UV photocure is carried out, is an attractive technique because the physical property of LC can be improved and furthermore the new characteristics may occur in terms of the coupling effect between LC molecules and polymer [6-14]. Furthermore, the polymer-stabilized (PS) LC devices have a great 
potential for flexible large-size LCDs because of the stabilization of the cell gap and the LC molecular alignment structure. In this paper, we mainly focus on the decay response time $\left(t_{\text {decay }}\right)$ from on-state to off-state of electric field because $t_{\text {decay }}$ cannot be controlled by electric field though the rise time $\left(t_{\text {rise }}\right)$ can be. We expect that a high-speed decay response can be obtained by the polymer stabilization of the initial off-state.

\section{Experimentals}

The materials used in this research were as follows: the nematic LC was MLC-2062 (Merck); the photocurable mesogenic acrylate monomer was monoacrylate UCL-001 (DIC) which was doped with $1 \mathrm{wt} \%$ photoinitiator; and the LC alignment film was polyimide SE-7492 (Nissan Chemical Industries).

We fabricated twisted nematic (TN) LC cells as follows. A solution of polyimide was spun on glass substrates coated with indium-tin oxide (ITO) and then baked. After the thermal treatment, the substrates were rubbed. Then, the nematic LC, which was doped with the photocurable mesogenic monomer, was injected in the isotropic phase via capillary action into an empty cell, in which the rubbing directions and cell gap were set perpendicular and $3 \mu \mathrm{m}$, respectively. Next, the cell was cooled gradually to the room temperature. After that, the LC medium was photocured with a UV light source $\left(365 \mathrm{~nm}, 10 \mathrm{~mW} / \mathrm{cm}^{2}\right)$. The $t_{\text {decay }}$ of TN-LC cell is well known as

$$
t_{\text {decay }}=\frac{\gamma d^{2}}{\pi^{2} K},
$$

where $\gamma$ is coefficient of viscosity, $d$ is cell gap and $K$ is modulus of elasticity. Thus, the $t_{\text {decay }}$ strongly depends on the cell gap, and therefore, we fabricated narrow-gap TN (NTN) LC cells with $3 \mu \mathrm{m}$ cell gap.

The electrooptical characteristics of PS-NTN-LC cells fabricated by above method were measured with a conventional measuring system based on a polarizing microscope. We defined the response time as follows: $t_{\text {rise }}$ and $t_{\text {decay }}$ are time for $90 \%$ transmittance variation at the on and off states, respectively, as shown in Fig. 3. And we also defined the threshold voltage as the voltage for 50\% transmittance variation, as shown in Fig. 4. We investigated the dependence of the electrooptical characteristics on the fabricating conditions of PS-LCs such as UV energy, polymer concentration, and temperature at photocure.

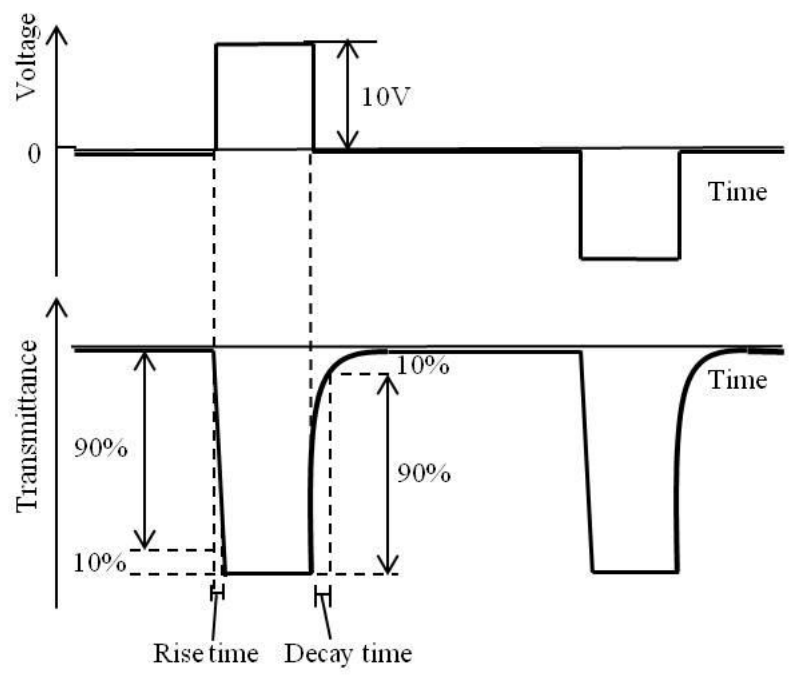

Fig. 3 Definition of response time.

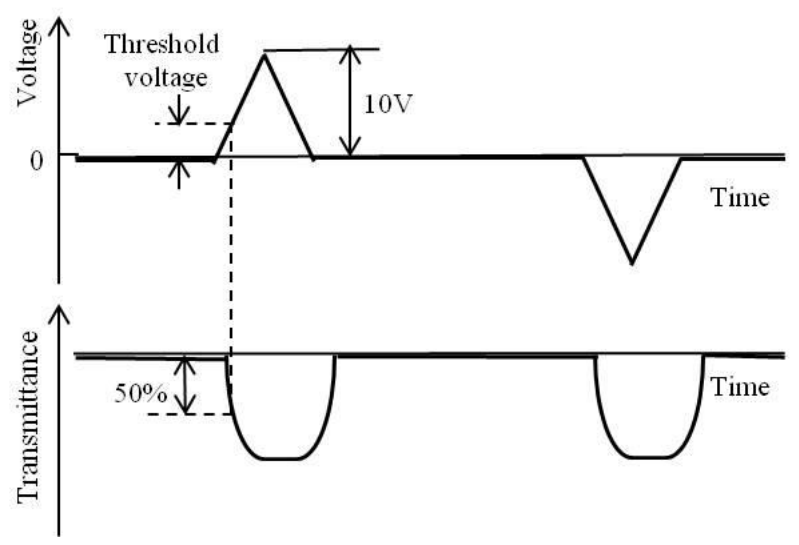

Fig. 4 Definition of threshold voltage.

\section{Results and discussion}

\subsection{UV irradiation energy}

Figure 5 shows the UV irradiation time dependence of $t_{\text {decay }}$ measured with a bipolar pulsed AC voltage (pulse width $1 \mathrm{~ms}$, amplitude $\pm 10 \mathrm{~V}$ and frequency $1 \mathrm{~Hz}$ ) at $30^{\circ} \mathrm{C}$. The PS-NTN-LC cell used was fabricated with $6 \mathrm{wt} \%$ monomer concentration and photocure at $30^{\circ} \mathrm{C}$. It is found that the response time of PS-LC takes a minimum at a certain UV energy. It is guessed that in the case of the long time UV irradiation, the molecular weight of the polymer may become large during the photocure and the polymer networks may exist bulky and locally. As a result, although the response of the LC molecules near the polymer can be fast, that far from the polymer remains slow. Therefore, the UV irradiation energy is a very important factor for realizing the high-speed response of PS-LC 


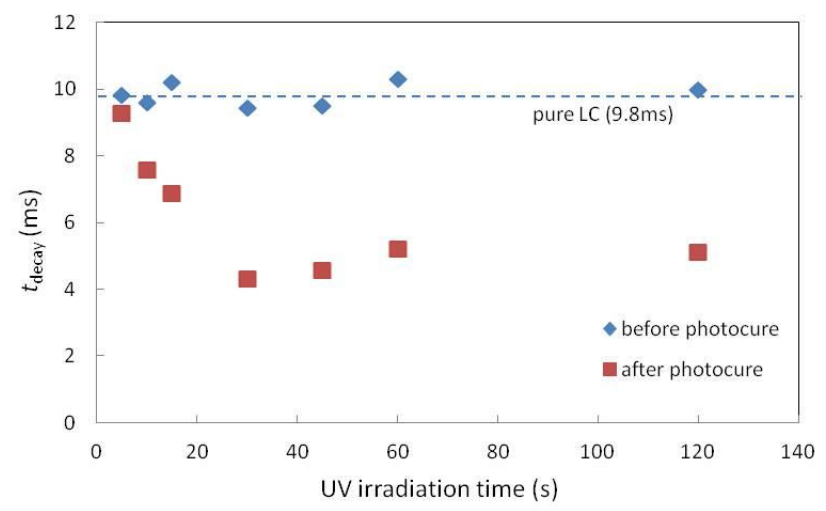

Fig. 5 UV irradiation time dependence of $t_{\text {decay. }}$.

\subsection{Polymer concentration}

Figure 6 shows the temperature dependence of $t_{\text {decay }}$ in the PS-NTN-LC cell fabricated by $30 \mathrm{~s}$ photocure at $30^{\circ} \mathrm{C}$. It is found that the higher the concentration of polymer is, the faster the response time is. It is thought that the polymer networks can strongly stabilize the initial off-state LC molecular alignment structure and then the high-speed decay response can be obtained.

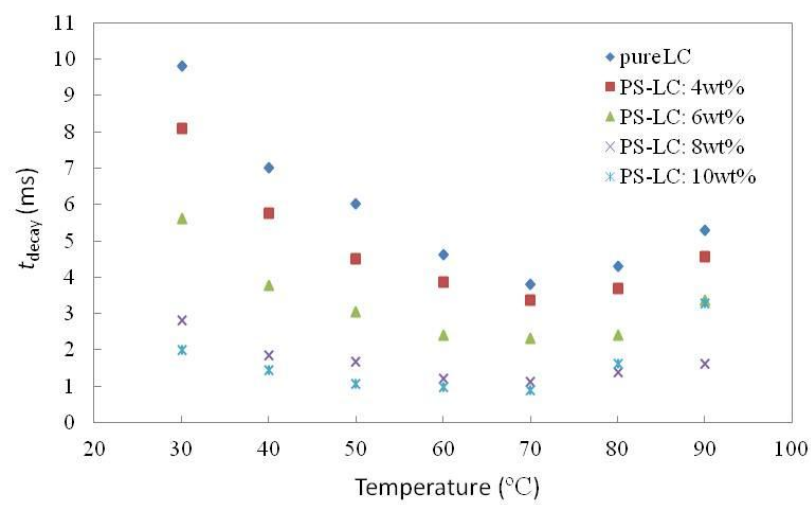

Fig. 6 Temperature dependence of $t_{\text {decay }}$.

On the other hand, it was found that the higher the concentration of polymer is, the higher the threshold voltage and $t_{\text {rise }}$ are, as shown in Fig. 7 and 8 , respectively. Since the polymer networks stabilize the initial off-state LC alignment, the threshold voltage and $t_{\text {rise }}$ may increase due to the polymer stabilization. Thus, it is found that there exists the trade-off relation between the $t_{\text {decay }}$ and the threshold voltage and $t_{\text {rise. }}$. Therefore, it is important to select a suitable polymer concentration for fabricating an available LCD devise.

\subsection{Temperature at photocure}

Table 1 shows the dependence of $t_{\text {decay }}$ on the temperature at photocure $\left(T_{\mathrm{UV}}\right)$ in the PS-NTN-LC

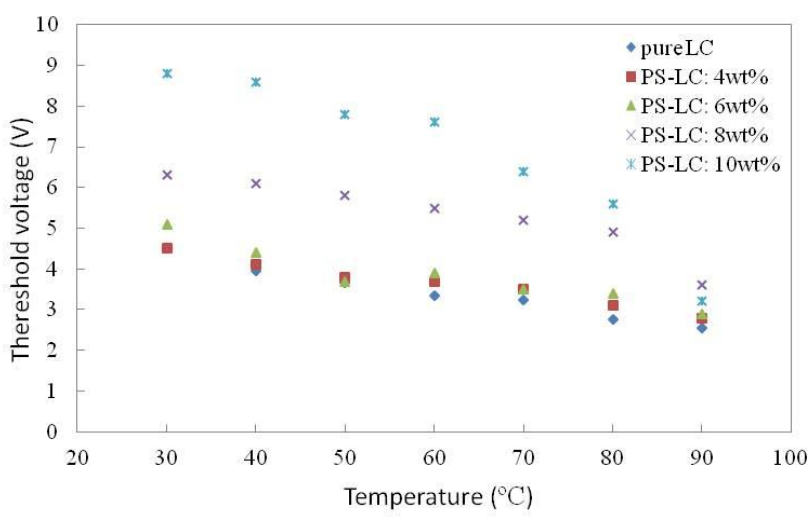

Fig. 7 Temperature dependence of threshold voltage.

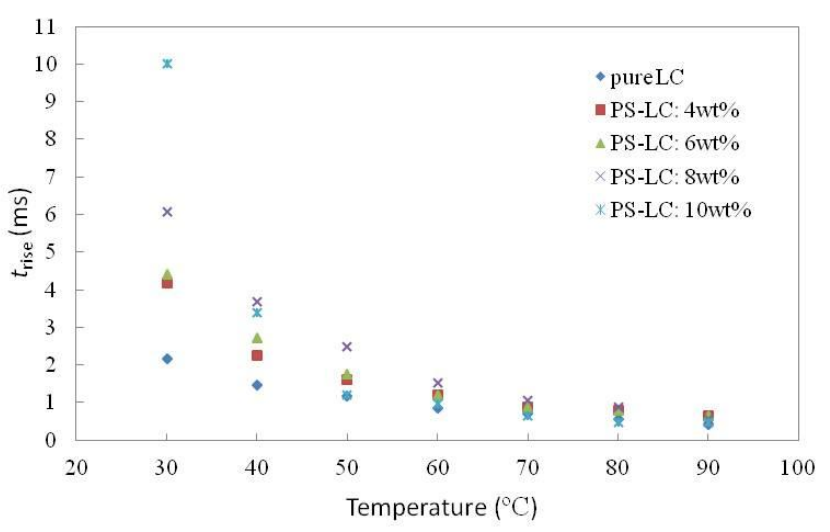

Fig. 8 Temperature dependence of $t_{\text {rise }}$.

fabricated with $6 \mathrm{wt} \%$ monomer concentration and $30 \mathrm{~s}$ photocure. The $t_{\text {decay }}$ was measured at $30^{\circ} \mathrm{C}$. It is found that the $t_{\text {decay }}$ of PS-LC strongly depends on $T_{\mathrm{UV}}$, and $t_{\text {decay }}$ increases as $T_{\mathrm{UV}}$ increases. It is guessed that in the high temperature, the high thermal motion of molecules appears and thus, the order parameter of LC medium lowers. The polymer networks formed at the high temperature may relatively lack in uniformity of the orientation and then have a distorted TN arrangement. As a result, the macroscopic strength of the polymer stabilization may decrease, and therefore, the decay response may be relatively slow. It is important to lowers the temperature at the photocure for lowering $t_{\text {decay }}$.

\section{Conclusions}

In this research, we tried to obtain a high-speed response of nematic liquid crystals by using polymer stabilization technique for realization of the FSC-LCDs. We researched the dependence of the electrooptical characteristics such as response time and threshold voltage on UV irradiation energy, polymer concentration and temperature at photocure. As a result, it was found 
Table 1 Dependence of $t_{\text {decay }}$ on temperature at photocure: $t_{\text {decay }}$ was measured at $30^{\circ} \mathrm{C}$.

\begin{tabular}{ccc}
\hline \multirow{2}{*}{$\begin{array}{c}\text { Temperature at } \\
\text { photocure } T_{\mathrm{UV}}\end{array}$} & \multicolumn{2}{c}{$t_{\text {decay }}(\mathrm{ms})$} \\
\cline { 2 - 3 }$\left({ }^{\circ} \mathrm{C}\right)$ & $\begin{array}{c}\text { before } \\
\text { photocure }\end{array}$ & $\begin{array}{c}\text { after } \\
\text { photocure }\end{array}$ \\
\hline 30 & 9.4 & 4.3 \\
70 & 9.1 & 7.9 \\
\hline
\end{tabular}

that the electrooptical characteristics strongly depend on their conditions. The $t_{\text {decay }}$ of PS-LC takes a minimum at a certain UV energy for photocuring. The $t_{\text {decay }}$ decreases as the polymer concentration increases, although the threshold voltage and $t_{\text {rise }}$ increases. And then, the $t_{\text {decay }}$ increases as $T_{\mathrm{Uv}}$ increases.

\section{Acknowledgements}

We gratefully thank Dr. H. Ichinose of Merck, Dr. H. Takatsu and Dr. H. Hasebe of DIC, and Dr. H. Fukuro and Mr. H. Endoh of Nissan Chem. Ind. for supplying materials used in this research.

\section{References}

[1] H. Hasebe and S. Kobayashi, SID Dig. Tech. Papers, 16 (1985) 81.

[2] T. Tanaka, H. Hasebe and S. Kobayashi, Proc. JPN Display'86, (1986) 360.
[3] T. Makino, Y. Kiyota, T. Yoshihara, H. Shiroto and H. Inoue, 1998 Liquid Crystal Symp. Jpn. Liquid Crystal Society, 3-3b (1998) 204.

[4] T. Uchida, K. Saitoh, T. Miyashita and M. Suzuki, Proc. IDRC '97, (1997) 37.

[5] T. Takahashi, H. Furue, M. Shikada, N. Matsuda, T. Miyama and S. Kobayashi, Jpn. J. Appl. Phys., 38 (1999) L534.

[6] H. Furue, T. Miyama, Y. Iimura, H. Hasebe, H. Takatsu and S. Kobayashi, Jpn. J. Appl. Phys., 36 (1997) L1517.

[7] M. Shikada, Y. Tanaka, J. Xu, K. Furuichi, H. Hasebe, H. Takatsu and S. Kobayashi, Jpn. J. Appl. Phys. 40 (2001) 5008.

[8] H. Furue, H. Yokoyama and S. Kobayashi, Jpn. J. Appl. Phys., 40 (2001) 5790.

[9] H. Furue, R. Uchino and J. Hatano, $J$. Photopolymer Sci. and Tech., 18 (2005) 33.

[10] H. Furue, H. Miyaura, and J.Hatano, $J$. Photopolym. Sci.Technol., 19 (2006) 163.

[11] H. Furue, K. Ikeda and Y. Yamazaki, J. Photopolym. Sci. Technol., 20 (2007) 19.

[12] H. Furue K. Ikeda, and Y. Yamazaki, Jpn. J. Appl. Phys., 46 (2007) 7132.

[13] D. Kakinuma, H. Kuramochi and H. Furue, $J$. Photopolym. Sci. Technl., 22 (2009) 537.

[14] H. Furue, K. Okazoe and M. Tamura, J. Photopolym. Sci.Technol., 23 (2010) 349. 